\title{
Noncollinear single-electron spin-valve transistors
}

\author{
Wouter Wetzels and Gerrit E. W. Bauer \\ Kavli Institute of Nanoscience, Delft University of Technology, Lorentzweg 1, 2628 CJ Delft, The Netherlands \\ Milena Grifoni \\ Institut für Theoretische Physik, Universität Regensburg, 93035 Regensburg, Germany
}

(Received 4 April 2005; published 19 July 2005)

\begin{abstract}
We study interaction effects on transport through a small metallic cluster connected to two ferromagnetic leads (a single-electron spin-valve transistor) in the "orthodox model" for the Coulomb blockade. The nonlocal exchange between the spin accumulation on the island and the ferromagnetic leads is shown to affect the transport properties such as the electric current and spin-transfer torque as a function of the magnetic configuration, gate voltage, and applied magnetic field.
\end{abstract}

DOI: 10.1103/PhysRevB.72.020407

PACS number(s): 85.75.-d, 72.25.Mk, 73.23.Hk

Magnetoelectronics is a contender to fulfill the technological need for faster and smaller memory and sensing devices. The drive into the nanometer regime brings about an increasing importance of electron-electron interaction effects. Small metallic clusters (islands) that are electrically contacted to metallic leads by tunnel junctions and capacitively coupled to a gate electrode can behave as "singleelectron transistors (SETs)." In the Coulomb-blockade regime, the charging energy needed to change the electron number on the island by one exceeds the thermal energy, and transport can be controlled on the level of the elementary charge. ${ }^{1}$ In a spin-valve SET (SV-SET), the contacts to the cluster consist of ferromagnetic metals $(F)$. We focus here on $\mathrm{F}|\mathrm{N}| \mathrm{F}$ structures with normal-metal (N) islands [see Fig. $1(\mathrm{a})]^{2}$ since these "spin valves" display giant magnetoresistance and spin-current-induced magnetization reversal. ${ }^{3}$ Other combinations such as $\mathrm{F}|\mathrm{F}| \mathrm{F}$ (Ref. 4), $\mathrm{N}|\mathrm{N}| \mathrm{F}$ (Ref. 5), or $\mathrm{F}|\mathrm{F}| \mathrm{N}$ (Refs. 6 and 7) are of interest as well.

Several theoretical studies have been devoted to the binary magnetoresistance (MR) of SV-SETs, i.e., the difference in the electric resistance between parallel and antiparallel configurations of the magnetization directions. ${ }^{8,9}$ Interaction effects in magnetic devices have been studied as well for spin valves with a Luttinger liquid island ${ }^{10}$ and for single-level quantum dots ${ }^{11-13}$ with noncollinear magnetic configurations.

A necessary condition for a significant $\mathrm{MR}$ in $\mathrm{F}|\mathrm{N}| \mathrm{F}$ structures is a spin accumulation on the normal-metal island, viz. a sufficiently long spin-flip relaxation time $\tau_{\mathrm{sf}}$. Seneor et al. ${ }^{14}$ have measured the MR of SV-SETs with gold islands with a $\tau_{\mathrm{sf}} \sim 800 \mathrm{ps}$. A $\tau_{\mathrm{sf}}$ in the microsecond regime has been reported for Co nanoclusters. ${ }^{6}$ The long spin-flip times in small clusters is not yet fully understood; it might simply be due to the probability of finding zero impurities in a given small cluster.

In this Rapid Communication we discuss aspects of electron transport in metallic SV-SETS with noncollinear magnetization directions. It turns out that an effective exchange effect between the spin accumulation and the magnetizations has to be taken into account.

We take the junction resistances sufficiently larger than the quantum resistance $R_{Q}=h / e^{2}$ so that the Coulomb block- ade can be treated by lowest-order perturbation theory. We furthermore disregard the size quantization of states in the clusters, thus adopting the well-established "orthodox" model. ${ }^{1}$ In our model system [cf. Fig. 1(b)], the ferromagnetic leads are treated as reservoirs with single-domain magnetization directions $\vec{m}_{1}$ and $\vec{m}_{2}$. Disregarding magnetic anisotropies, the relevant parameter is the angle $\theta$ between the magnetizations. The capacitances of the junctions are $C_{1}$ and $C_{2}$. The cluster is capacitively coupled to the gate, with capacitance $C_{G} \ll C_{1}, C_{2}$.

We assume a separation of time scales between the energy relaxation that rapidly thermalizes injected charges and the slow spin relaxation. In this regime, the quasiequilibrium excess spin $\vec{s}$ on the normal-metal island is well defined. In second quantization $\vec{s}=(\hbar / 2) \sum_{k s s^{\prime}}\left\langle c_{k s}^{\dagger} \vec{\sigma}_{s s^{\prime}} c_{k s^{\prime}}\right\rangle$ where $\vec{\sigma}$ is the vector of Pauli spin matrices and $k, s$ denote the orbital and spin indices of the island states, respectively. This corresponds to a chemical potential difference (spin accumulation) $\Delta \mu=2 \delta|\vec{s}| / \hbar$, where $\delta$ is the average single-particle energy separation [in terms of the static susceptibility $\chi_{s}$ : $\left.\Delta \mu=2 \mu_{B}^{2}|\vec{s}| /\left(\chi_{s} \hbar\right)\right]$. Spin-flip relaxation is parametrized by the spin-flip time $\tau_{s f}$ or spin-flip conductance $G_{s f} \equiv e^{2} /\left(2 \tau_{s f} \delta\right)$. For metals, $\delta$ is much smaller than the thermal energy except for very small particles (diam $\lesssim 5 \mathrm{~nm}$ ). We restrict our attention here to a regime in which the bias energy is small compared to the thermal and the charging energies, but large compared to $\delta$, so that the transport properties do not depend on the energy-relaxation rate. ${ }^{9}$ The island state is then characterized by the excess number of electrons $n$ and net spin angular momentum $\vec{s}$. A state

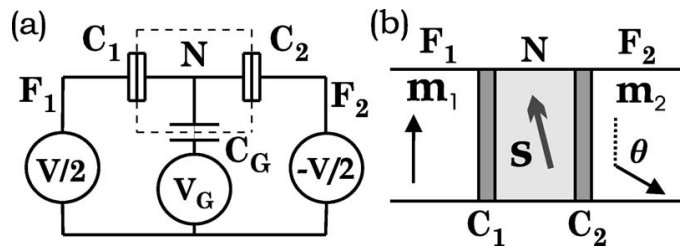

FIG. 1. (a) The spin-valve single-electron transistor. (b) The tunneling rates between the leads and the cluster depend on the spin accumulation $\vec{s}$. 
distribution on the island governed by free-energy minimization under these spin and charge constraints can be used.

The master equation for electron transport in the orthodox model is determined by a tunneling Hamiltonian that can be treated by perturbation theory. We derive the appropriate Hamiltonian by collecting the leading terms in the transition probabilities. A crucial parameter is the mixing conductance of an $\mathrm{N} \mid \mathrm{F}$ junction $^{15}$

$$
G^{\uparrow \downarrow} \equiv \frac{e^{2}}{h} \sum_{n m}\left(\delta_{m n}-r_{\uparrow}^{n m}\left(r_{\downarrow}^{n m}\right)^{*}\right),
$$

where $n$ and $m$ denote the transport channels in the normal metal, and $r_{\uparrow}^{n m}$ and $r_{\downarrow}^{n m}$ are the corresponding spin-dependent reflection coefficients. The real part of the mixing conductance determines the spin-transfer torque that strives to align the magnetization to the spin accumulation. ${ }^{3}$ In the limit of a tunneling contact, $\operatorname{Re} G^{\uparrow \downarrow}=\left(G_{\uparrow}+G_{\downarrow}\right) / 2$, where $G_{s}=\left(e^{2} / h\right) \Sigma_{n m}\left(\delta_{m n}-\left|r_{s}^{n m}\right|^{2}\right)$ is the conventional tunneling conductance for spin $s$. The torque is transferred by the electrons that tunnel through the contact and is included in the standard spin-dependent tunneling Hamiltonian. The imaginary part of the mixing conductance can be interpreted as an effective field parallel to the magnetization direction of the ferromagnet. For a normal metal separated from a Stoner ferromagnet by a specular, rectangular barrier we can directly solve the Schrödinger equation. With Fermi momenta of the ferromagnet of $k_{F \uparrow}=1.09 \AA^{-1}, k_{F \downarrow}=0.42 \AA^{-1}$ (characteristic for Fe), a normal-metal Fermi energy of $\epsilon_{F}=2.6 \mathrm{eV}$, a barrier height of $3 \mathrm{eV}$, and free-electron masses (cf. Ref. 3) we find $\operatorname{Im} G^{\uparrow \downarrow} /\left(G_{\uparrow}+G_{\downarrow}\right)=-0.26$. This illustrates that, in contrast to metallic interfaces, ${ }^{16}$ the imaginary part of the mixing conductance can be significant for tunnel junctions. This result is not sensitive to the width of the barrier but may depend strongly on material combination and interface morphology. For tunneling barriers made from magnetic insulators, ${ }^{17} \operatorname{Re} G^{\uparrow \downarrow}$ and $\operatorname{Im} G^{\uparrow \downarrow}$ may become large compared to $\left(G_{\uparrow}+G_{\downarrow}\right)$, which results in different physics. ${ }^{18}$

The Hamiltonian for the SV-SET reads

$$
H=H_{N}+\sum_{\alpha=1,2}\left(H_{F \alpha}+H_{T \alpha}+H_{\mathrm{ex} \alpha}\right),
$$

where $H_{N}$ describes the electrons on the normal metal and includes the electrostatic interaction energy ${ }^{19}$

$$
H_{N}=\sum_{k s} \varepsilon_{k} c_{k s}^{\dagger} c_{k s}+\frac{e^{2}\left(n-C_{G} V_{G} / e\right)^{2}}{2\left(C_{1}+C_{2}\right)} .
$$

For the two ferromagnetic leads $(\alpha=1,2)$ $H_{F \alpha}=\Sigma_{k s} \varepsilon_{\alpha k s} a_{\alpha k s}^{\dagger} a_{\alpha k s}$ and $n$ denotes the excess electron number on the island. The tunneling Hamiltonian for each contact reads $H_{T \alpha}=\Sigma_{k q s} T_{\alpha k q s} a_{\alpha k s}^{\dagger} c_{\alpha q s}+$ h.c.. Finally, the imaginary part of the mixing conductance gives rise to an effective exchange effect,

$$
H_{\mathrm{ex} \alpha}=\sum_{k s s^{\prime}} \Delta_{\mathrm{ex} \alpha} \vec{m}_{\alpha} \cdot c_{k s}^{\dagger} \vec{\sigma}_{s s^{\prime}} c_{k s^{\prime}},
$$

where $\Delta_{\mathrm{ex} \alpha}=-\hbar \delta \operatorname{Im} G_{\alpha}^{\uparrow \downarrow} /\left(2 e^{2}\right)$. We note that the effect of Eq. (4) on the spin accumulation is identical to that of an external magnetic field applied in the direction $\vec{m}_{\alpha}$. Such an effective exchange Hamiltonian has been introduced earlier for a Luttinger liquid attached to ferromagnetic leads. ${ }^{10}$ Physically, electrons in the normal-metal island feel the ferromagnet through their tunneling tails that cause a spin dependence of the reflection coefficients. The small spin splitting due to $\Delta_{\mathrm{ex}}$ in the ground state does not influence the transport to leading order in the tunneling probability.

Recently, the angular dependence of transport through spin valves has been studied for single-level quantum dot islands. ${ }^{11,12}$ In these systems an effective field was found to act on unpaired quantum dot electron spins that is caused by virtual particle exchange with the leads in the Coulomb blockade. The same effect was discussed for few-level quantum dots in Ref. 13. These correlations cause effects similar to those discussed here, but their physical origin is completely different. Equation (4) is caused by electron exchange on a fast time scale corresponding to the reciprocal Fermi energy and reflects the electronic band structure of the junctions, independent of the applied voltages and charging energies. In contrast, the correlation-mediated exchange is induced on time scales of the reciprocal charging energy, changes sign with gate voltage, and does not vanish for normal-metal contacts. ${ }^{13}$ Correlation exchange can be taken into account by an additional gate-voltage-dependent effective magnetic field, parallel to the magnetization direction of the ferromagnet. We find that also for classical islands the correlation exchange field can be of the same order as $\Delta_{\mathrm{ex}}$, when the tunneling conductances and $\operatorname{Im} G^{\uparrow \downarrow}$ are of comparable size. Since both effects can at least in principle be distinguished experimentally by gate voltage and temperature dependence, a more detailed discussion is deferred to a future publication.

We introduce spin-dependent conductances for both junctions, $G_{\alpha s} \equiv \pi e^{2} \rho_{N} \rho_{F \alpha s} \bar{T}_{\alpha s} / \hbar \quad(\alpha=1,2, s=\uparrow, \downarrow) . \quad \rho_{N}$ is the density of states at the Fermi level in the normal metal, and $\rho_{F \alpha s}$ the spin-dependent density of states in ferromagnet $\alpha$. $\bar{T}_{s}$ is proportional to the average tunneling probability over all channels for spin $s, \bar{T}_{s} \equiv\left\langle\left|T_{m n s}\right|^{2}\right\rangle_{m n}$. The conductances are assumed to be constant within the energy interval of the charging energy, which is a safe assumption for metals. We introduce the total conductances $G_{\alpha} \equiv G_{\alpha \uparrow}+G_{\alpha \downarrow}$, the polarizations $P_{\alpha} \equiv\left(G_{\alpha \uparrow}-G_{\alpha \downarrow}\right) / G_{\alpha}$, and $F(E) \equiv E /\left(1-e^{-\beta E}\right)$. The tunneling rate for adding an electron through contact $\alpha(=1,2=+,-)$ in the considered regime where $e V \ll k_{B} T$ then reads

$$
\begin{aligned}
\Gamma_{\alpha}^{n \rightarrow n+1}(V, q, \vec{s})= & \frac{G_{\alpha}}{e^{2}} F\left(-E_{\alpha}(V, q)\right) \\
& -\frac{G_{\alpha}}{e^{2}} F^{\prime}\left(-E_{\alpha}(V, q)\right) \frac{P_{\alpha} \Delta \mu}{2}\left(\vec{m}_{\alpha} \cdot \hat{s}\right),
\end{aligned}
$$

where $E_{\alpha}(V, q)=\alpha \kappa_{\alpha} e V-e(q-e / 2) /\left(C_{1}+C_{2}\right)$ is the electrostatic energy difference associated with the tunneling of one electron into the cluster from lead $\alpha$ with $q=-n e+C_{G} V_{G}$ the charge on the island and $\kappa_{\alpha} \equiv\left(1 / C_{1}+1 / C_{2}\right)^{-1} / C_{\alpha}$. The other rates can be found analogously. We can also find the net spin current into the cluster due to tunneling events in which an 

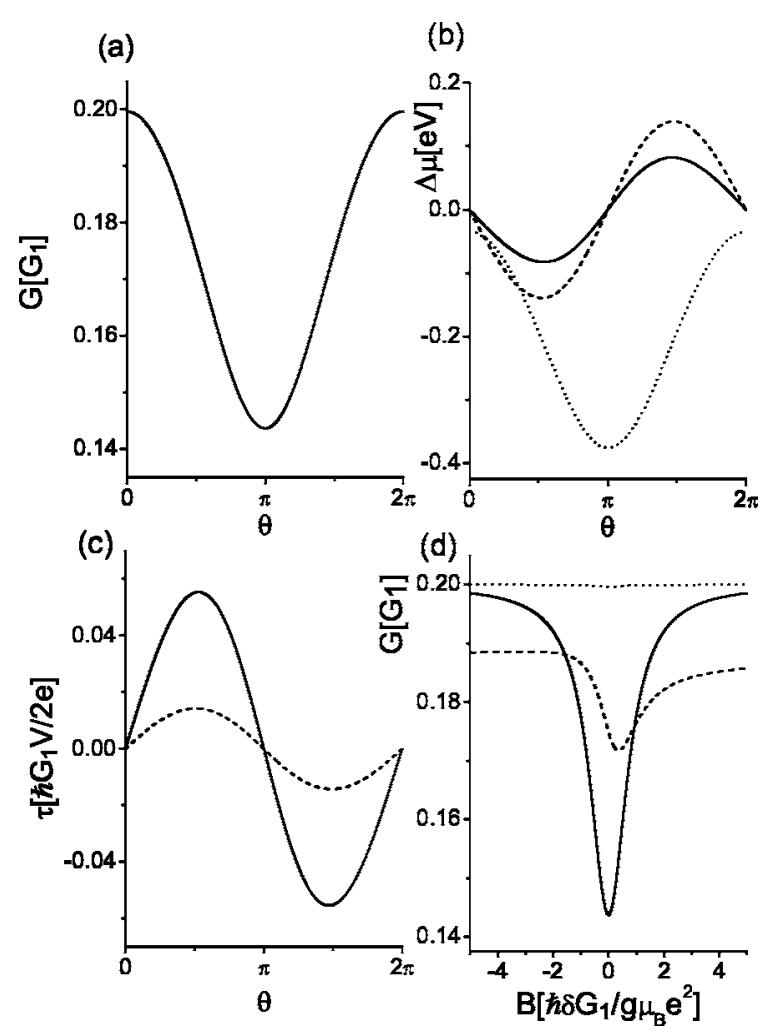

FIG. 2. Results for a SV-SET with $G_{2}=2 G_{1} / 3, \quad P_{1}=0.8$, $P_{2}=0.7, G_{s f}=G_{1} / 5, \operatorname{Im} G_{1}^{\uparrow \downarrow}=G_{1} / 4, \operatorname{Im} G_{2}^{\uparrow \downarrow}=G_{2} / 4$, and $\Delta_{\min }=0$. (a) Conductance as a function of the angle between the magnetizations of the leads. (b) Spin accumulation components in the direction of $\vec{m}_{1}$ (dotted), $\vec{z} \times \vec{m}_{1}$ (dashed), and $\vec{z}$ (solid). (c) Spin-transfer torque on ferromagnet 1 in the direction $\vec{z} \times \vec{m}_{1}$ (dashed) and $\vec{z}$ (solid). (d) Conductance vs magnetic field applied in the direction $\vec{z} \times \vec{m}_{1}$ for $\theta=0$ (dotted), $\theta=\pi / 2$ (dashed), and $\theta=\pi$ (solid).

electron is added to the island through contact $\alpha$,

$$
\begin{aligned}
\left(\frac{d \vec{s}}{d t}\right)_{\alpha, \text { tun }}^{n \rightarrow n+1}= & \frac{\hbar}{2} \frac{G_{\alpha}}{e^{2}} F\left(-E_{\alpha}(V, q)\right) P_{\alpha} \vec{m}_{\alpha} \\
& -\frac{\hbar}{2} \frac{G_{\alpha}}{e^{2}} F^{\prime}\left(-E_{\alpha}(V, q)\right) \frac{\Delta \mu}{2} \hat{s} .
\end{aligned}
$$

The dynamics of the spin accumulation is also affected by spin-flip scattering, the exchange effect discussed above, and an external magnetic field $\vec{B}$,

$$
\begin{gathered}
\left(\frac{d \vec{s}}{d t}\right)_{\mathrm{sf}}=-\vec{s} / \tau_{s f}, \\
\left(\frac{d \vec{s}}{d t}\right)_{\mathrm{ex}}=\sum_{\alpha} \frac{\operatorname{Im} G_{\alpha}^{\uparrow \downarrow} \delta}{e^{2}}\left(\vec{m}_{\alpha} \times \vec{s}\right), \\
\left(\frac{d \vec{s}}{d t}\right)_{\text {magn }}=\frac{g \mu_{B}}{\hbar}(\vec{B} \times \vec{s}) .
\end{gathered}
$$

The master equation for the SV-SET is $d p_{n} / d t$ $=-p_{n}\left(\Gamma^{n \rightarrow n+1}+\Gamma^{n \rightarrow n-1}\right)+p_{n+1} \Gamma^{n+1 \rightarrow n}+p_{n-1} \Gamma^{n-1 \rightarrow n}$ combined with $d \vec{s} / d t$ described above. Here, $p_{n}$ is the probability dis-

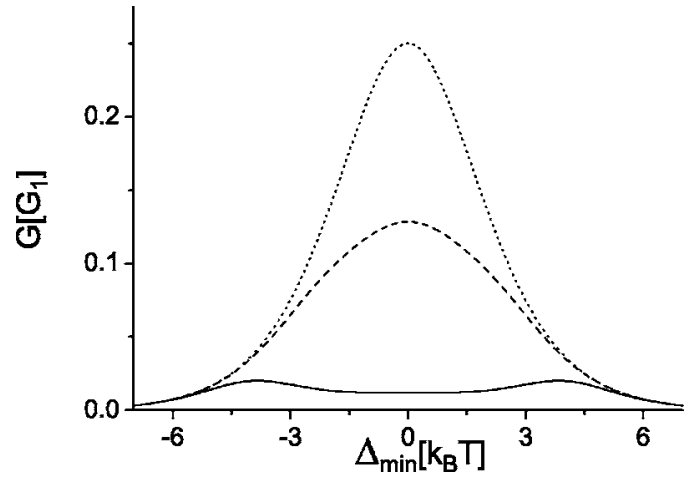

FIG. 3. Conductance $(G)$ as a function of gate voltage for $\theta=0$ (dotted), $\theta=0.6 \pi$ (dashed), and $\theta=0.9 \pi$ (solid). The ferromagnets are half-metallic and the conductance of both contacts is $G_{1}$. $\operatorname{Im} G_{1}^{\uparrow \downarrow}=\operatorname{Im} G_{2}^{\uparrow \downarrow}=G_{1} / 4, G_{s f}=0$.

tribution for the number of electrons on the island and $\Gamma^{n \rightarrow n+1}$ denotes the rate for adding another electron when the cluster has $n$ excess electrons. We now focus on a quasistationary state $d p_{n} / d t=0$. When $e V \ll k_{B} T \ll e^{2} / 2 C$ the number of electrons on the island fluctuates between two values denoted by $n=$ " 0 " and " 1. " We can use detailed balance symmetry to find the stationary state, whence $p_{0} \Gamma^{0 \rightarrow 1}=p_{1} \Gamma^{1 \rightarrow 0}$. We find the conductance $G$,

$$
G\left(V_{G}, \vec{s}\right)=G_{\mathrm{KS}}\left(V_{G}\right)\left[1+\frac{\Delta \mu}{2 e V}\left(P_{1} \vec{m}_{1}-P_{2} \vec{m}_{2}\right) \cdot \vec{s}|| \vec{s} \mid\right],
$$

where $G_{\mathrm{KS}}\left(V_{G}\right) \equiv \beta \Delta_{\min } G_{1} G_{2} /\left[2\left(G_{1}+G_{2}\right) \sinh \beta \Delta_{\text {min }}\right]$ with $\Delta_{\text {min }} \equiv e\left(C_{G} V_{G}-e / 2\right) /\left(C_{1}+C_{2}\right)$ describes one Coulombblockade oscillation. ${ }^{20}$

The spin accumulation is obtained from the condition $d \vec{s} / d t=0$. For a symmetric setup, in which the conductance parameters that characterize the two contacts are equal, we find

$$
\frac{G\left(V_{G}\right)}{G_{\mathrm{KS}}\left(V_{G}\right)}=1-\frac{P^{2}}{1+\frac{G_{s f}}{G_{\mathrm{KS}}\left(V_{G}\right)}} \frac{\tan ^{2} \frac{\theta}{2}}{\tan ^{2} \frac{\theta}{2}+1+\left[\frac{\operatorname{Im} G^{\uparrow \downarrow}}{G_{\mathrm{KS}}\left(V_{G}\right)+G_{s f}}\right]^{2}} .
$$

When the imaginary part of the mixing conductance vanishes, the angular dependence of the conductance is proportional to a cosine. The result of Brataas et al. for a $\mathrm{F}|\mathrm{N}| \mathrm{F}$ spin valve without interaction ${ }^{15}$ is recovered by substituting $\beta \Delta_{\text {min }} /\left(2 \sinh \beta \Delta_{\text {min }}\right)$ by 1 (cf. Ref. 9).

In Fig. 2(a) an example of the dependence of the conductance on the angle $\theta$ is shown. When the magnetizations are noncollinear, the exchange effect reduces the spin accumulation and increases the conductance. In Fig. 2(b), the spin accumulation as a function of angle illustrates that for noncollinear magnetizations, the nonlocal exchange pulls the spin accumulation vector out of the plane of the magnetizations. 
The spin current between the ferromagnets and the normal metal gives rise to a spin-transfer torque $\vec{\tau}_{\alpha}=-\vec{m}_{\alpha} \times\left(\mathbf{I}_{s}\right.$ $\times \vec{m}_{\alpha}$ ) on ferromagnet $\alpha$ [see Fig. 2(c)]. $\mathbf{I}_{s}$, the net spin flow out of the ferromagnet, is strongly modulated by the gate voltage. When the Coulomb interaction suppresses the current, the exchange effect becomes relatively more important. Figure 2(d) shows that, for noncollinear configurations, the exchange effect causes an asymmetry in the Hanle effect with respect to the sign of an applied external magnetic field.

The curves in Fig. 3 show the gate-voltage modulated conductance for a symmetric spin valve with half-metallic ferromagnetic leads $(P=1)$. Spin flip is disregarded. When the magnetizations are parallel $(\theta=0)$, no spin accumulates on the island and the Coulomb-blockade oscillation equals that of normal-metal systems. When the angle $\theta$ is increased, the conductance is suppressed by a counteracting spin accumulation. The exchange effect acts to reduce this spin accumulation for noncollinear configurations and can cause a local conductance minimum at $\Delta_{\min }=0$ (e.g., at $\theta=0.9 \pi)$. Deep in the Coulomb blockade, a significant spin accumulation is prevented from building up and all curves converge.

Interestingly, the angular magnetoresistance for Luttinger liquids with ferromagnetic contacts ${ }^{10}$ looks very similar. In order to find experimental evidence for spin-charge separation it is therefore necessary to avoid spurious effects caused by the Coulomb blockade.

In conclusion, we studied the transport characteristics for noncollinear spin valves in the Coulomb-blockade regime. A nonlocal exchange interaction between the spin accumulation and the ferromagnets affects the conductance and spintransfer torque as a function of the gate voltage. This might provide possibilities to control charge and spin transport in nanoscale magnetoelectronic devices.

We acknowledge valuable discussions with Yuli Nazarov, Jan Martinek, Jürgen König, and Yaroslav Tserkovnyak. This research was supported by the NWO, FOM, and EU Commission FP6 NMP-3 project 05587-1 "SFINX."
${ }^{1}$ Single Charge Tunneling, edited by H. Grabert and M. H. Devoret (Plenum, New York, 1992).

${ }^{2}$ S. Sahoo, T. Kontos, C. Schönenberger, and C. Sürgers, Appl. Phys. Lett. 86, 112109 (2005); J. Philip, D. Wang, M. Muenzenberg, P. LeClair, B. Diouf, J. S. Moodera, and J. G. Lu, J. Magn. Magn. Mater. 272-276, 1949 (2004); M. Zaffalon and B. J. van Wees, Phys. Rev. Lett. 91, 186601 (2003); A. N. Pasupathy, R. C. Bialczak, J. Martinek, J. E. Grose, L. A. K. Donev, P. L. McEuen, and D. C. Ralph, Science 306, 86 (2004); L. Y. Zhang, C. Y. Wang, Y. G. Wei, X. Y. Liu, and D. Davidović, cond-mat/0502181 (unpublished).

${ }^{3}$ J. C. Slonczewski, Phys. Rev. B 39, 6995 (1989).

${ }^{4}$ K. Ono, H. Shimada, and Y. Ootuka, J. Phys. Soc. Jpn. 66, 1261 (1997); L. F. Schelp, A. Fert, F. Fettar, P. Holody, S. F. Lee, J. L. Maurice, F. Petroff, and A. Vaurès, Phys. Rev. B 56, R5747 (1997).

${ }^{5}$ M. M. Deshmukh and D. C. Ralph, Phys. Rev. Lett. 89, 266803 (2002)

${ }^{6}$ K. Yakushiji, F. Ernult, H. Imamura, K. Yamane, S. Mitani, K. Takanashi, S. Takahashi, S. Maekawa, and H. Fujimori, Nat. Mater. 4, 57 (2005).

${ }^{7}$ X. Waintal and O. Parcollet, Phys. Rev. Lett. 94, 247206 (2005).

${ }^{8}$ J. Barnaś and A. Fert, Phys. Rev. Lett. 80, 1058 (1998); K. Majumdar and S. Hershfield, Phys. Rev. B 57, 11521 (1998); A. N. Korotkov and V. I. Safarov, ibid. 59, 89 (1999); A. Brataas, Yu. V. Nazarov, J. Inoue, and G. E. W. Bauer, Eur. Phys. J. B 9, 421 (1999).

${ }^{9}$ A. Brataas and X. H. Wang, Phys. Rev. B 64, 104434 (2001).

${ }^{10}$ L. Balents and R. Egger, Phys. Rev. B 64, 035310 (2001); C.
Bena and L. Balents, ibid. 65, 115108 (2002).

${ }^{11}$ J. König and J. Martinek, Phys. Rev. Lett. 90, 166602 (2003); M. Braun, J. König, and J. Martinek, Phys. Rev. B 70, 195345 (2004); J. König, J. Martinek, J. Barnaś, and G. Schön, in CFN Lectures on Functional Nanostructures, edited by K. Busch et al., Lecture Notes in Physics Vol. 658 (Springer, Berlin, 2005), pp. 145-164.

${ }^{12}$ W. Rudziński, J. Barnaś, R. Świrkowicz, and M. Wilczyński, Phys. Rev. B 71, 205307 (2005); J. N. Pedersen, J. Q. Thomassen, and K. Flensberg, cond-mat/0412145 (unpublished); L. Y. Gorelik, S. I. Kulinich, R. I. Shekhter, M. Jonson, and V. M. Vinokur, cond-mat/0502243 (unpublished). J. Fransson, Europhys. Lett. 70, 796 (2005).

${ }^{13}$ S. Braig and P. W. Brouwer, Phys. Rev. B 71, 195324 (2005).

${ }^{14} \mathrm{P}$. Seneor et al. (private communication).

${ }^{15}$ A. Brataas, Yu. V. Nazarov, and G. E. W. Bauer, Phys. Rev. Lett. 84, 2481 (2000); A. Brataas, Y. V. Nazarov, and G. E. W. Bauer, Eur. Phys. J. B 22, 99 (2001).

${ }^{16}$ K. Xia, P. J. Kelly, G. E. W. Bauer, A. Brataas, and I. Turek, Phys. Rev. B 65, 220401(R) (2002).

${ }^{17}$ T. S. Santos and J. S. Moodera, Phys. Rev. B 69, 241203(R) (2004).

${ }^{18}$ D. Huertas-Hernando, Yu. V. Nazarov, and W. Belzig, Phys. Rev. Lett. 88, 047003 (2002); D. Huertas-Hernando and Yu. V. Nazarov, Eur. Phys. J. B 44, 373 (2005).

${ }^{19}$ Stricly speaking $n=\Sigma_{k s} c_{k s}^{\dagger} c_{k s}$ is here an operator.

${ }^{20}$ I. O. Kulik and R. I. Shekhter, Zh. Eksp. Teor. Fiz. 68, 623 (1975). 